\title{
Preparation and Characterization of Copper Oxide -Water Based Nanofluids by One Step Method for Heat Transfer Applications
}

\author{
K. SHREE MEENAKSHI*¹ and E. PRADEEP JAYA SUDHAN ${ }^{2}$ \\ ${ }^{1}$ Department of Chemistry, Loyola-ICAM College of Engineering and Technology, Chennai, \\ Tamil Nadu, India \\ ${ }^{2}$ Department of Chemistry, Anna University, Chennai, Tamil Nadu, India \\ shreemeenakshik@gmail.com
}

Received 28 October 2014 / Accepted 8 November 2014

\begin{abstract}
The aim of the current research is to manufacture copper oxide- water based nanofluids and to study its various properties. The copper oxide nanofluids were synthesized using water as the base fluid for potential use as a coolant for heat transfer applications. Three different concentrations $1 \%, 3 \%$ and $5 \%$ of the copper oxide nanofluids were developed. The characterization of the synthesized nanofluids was done by Fourier transform infrared spectroscopy (FT-IR), transmission electron microscope (TEM), energy dispersive spectroscopy (EDS) and x-ray diffraction (XRD) techniques. The thermo-physical properties (thermal conductivity and viscosity) measurements were carried out and the interesting results obtained are discussed.
\end{abstract}

Keywords: Nanofluids, Coolant, Thermo-physical properties, Energy dispersive spectroscopy

\section{Introduction}

Nanofluid is a novel heat transfer fluid prepared by dispersing nanometer-sized solid particles in traditional heat transfer fluid such as water or ethylene glycol to increase thermal conductivity and therefore heat transfer performance ${ }^{1-3}$. For example, when 0.3 volume percent of copper nano-particles are dispersed in ethylene glycol, one can observe about $40 \%$ of increase in thermal conductivity. Metal oxides such as aluminum oxide or titanium oxide are also feasible even though the amount of heat transfer increase is not as large as metal particles ${ }^{4-5}$.

The effectiveness of heat transfer enhancement is known to be dependent on the amount of dispersed particles, material type, particle shape, and so on. It is expected that nanofluid can be utilized in airplanes, cars, micro reactors among others. Before the introduction of nanofluid, it was expected that heat transfer could be enhanced by dispersing micron-sized particles. But the fluid with micron-sized particles caused problems due to sedimentation and clogging. Most of all, the fluid with micron sized particles was found to be not efficient 
enough. Since the concept of nanofluid has been first introduced, there have been many efforts to understand the mechanism of heat transfer enhancement together with experimental measurements of the thermal conductivity of nanofluids and the methods of utilization of nanofluids ${ }^{6}$.

In our current research we have synthesized copper oxide nanofluids by using water as the base fluid. The method is a simple one, less time consuming and ultimately the production cost may be reduced as well. These aspects of this work are novel.

\section{Experimental}

$\mathrm{CuO}$ nanoparticles of $25 \mathrm{~nm}$ (Sigma-Aldrich, USA) were dispersed in distilled water at $10 \mathrm{mM}$ concentration. Copper oxide nanofluids of concentration of $1 \%$, 3\% and $5 \%$ were prepared by mixing $1 \mathrm{~g}, 3 \mathrm{~g}$ and $5 \mathrm{~g}$ of nanoparticles in $100 \mathrm{~mL}$ of distilled water. To ensure proper mixing of the nanoparticles in water, sonication was performed for 30 min using bathsonicator equipment (37 kHz, $150 \mathrm{~W}$; TOSHCON Ultrasonic Cleaner, TPI Pvt. Ltd). Concentrations (in terms of the particles) were calculated using the average particle size and the bulk density of $\mathrm{CuO}$. The $\mathrm{pH}$ of the nanofluids was around 4.8 and they were clear transparent fluids.

Characterization of the synthesized copper oxide nanofluid was done by FT-IR, TEM, EDS and XRD. Thermo-physical properties (thermal conductivity and viscosity) measurements were carried out.

\section{Fourier transform infra-red spectroscopy (FT-IR)}

FTIR spectra were recorded using Perkin Elmer 781 infrared spectrometer with KBr pellets. The copper oxide nanofluid samples were directly applied by dabbing onto a $\mathrm{KBr}$ pellet.

\section{Transmission electron microscopy (TEM)}

TEM measurements of all the samples were done after they were drop-casted onto a carboncoated copper grid and dried in ambient conditions with a JEM 3011 (JEOL Ltd) $300 \mathrm{kV}$ high-resolution transmission electron microscope (HRTEM) with a UHR pole piece.

\section{Energy dispersive $X$-ray spectroscopy (EDS)}

Energy dispersive $\mathrm{x}$-ray spectroscopy (EDS) is an analytical technique used for the elemental analysis or chemical characterization of a sample. Its characterization capabilities are due in large part to the fundamental principle that each element has a unique atomic structure allowing x-rays that are characteristic of an element's atomic structure to be identified uniquely from one another.

\section{$X$-ray diffraction topography $(X R D)$}

Copper oxide nanofluid was diluted with absolute ethanol followed by centrifugation at $4000 \mathrm{rpm}$ for $60 \mathrm{~min}$. It was then washed with absolute ethanol and acetone. Further it was vacuum dried at $80{ }^{\circ} \mathrm{C}$ for $2 \mathrm{~h}$. X-ray diffraction topography of the obtained powder was performed on a X-ray Diffraction (XRD, MACMXP18) machine with wavelength: $1.54 \mathrm{~nm}$.

\section{Measurement of thermal conductivity}

For the measurement of Thermal conductivity, KD2 Pro was used. Different sensors were used for the measurement of these properties. As nanofluids are relatively low viscosity fluid, so $\mathrm{KS}^{-1}$ sensor was used to measure the thermal conductivity. These properties can be measured at various ranges of temperatures such as from $20^{\circ} \mathrm{C}$ to $80^{\circ} \mathrm{C}$. 


\section{Measurement of viscosity}

For the measurement of viscosity of nanofluids, Ostwald viscometer was used. This was done at various temperature ranges i.e. $20^{\circ} \mathrm{C}$ to $80^{\circ} \mathrm{C}$. Firstly, it was done for distilled water as distilled water is used as a base fluid for preparing nanofluids. Then, same method is done for the other samples i.e. $1 \%$, 3\% and 5\% volume concentrations of nanofluids and noted down the time at different temperatures.

\section{Results and Discussion}

\section{FT-IR spectra}

The FT-IR spectra of copper oxide nanofluid prepared in water is shown in Figure 1. The spectrum has absorption bands at about $3400 \mathrm{~cm}^{-1}$ and $1625 \mathrm{~cm}^{-1}$ were due to the stretching and bending vibrations of $-\mathrm{OH}$ group. In the region $550 \mathrm{~cm}^{-1}$ the signature band of $\mathrm{Cu}-\mathrm{O}$ vibration was observed. Further confirmation of the structure was done by EDAX.

\section{Transmission electron microscopy (TEM)}

TEM was used to further examine the particle size, crystallinity and morphology of samples. Analysis of the $\mathrm{CuO}$ nanoparticles by TEM showed the average diameter to be $20 \mathrm{~nm}$ (Figure 2). TEM bright field images of CuO show mostly spherical morphology. The TEM results are found to be agreement with the results of XRD analysis.

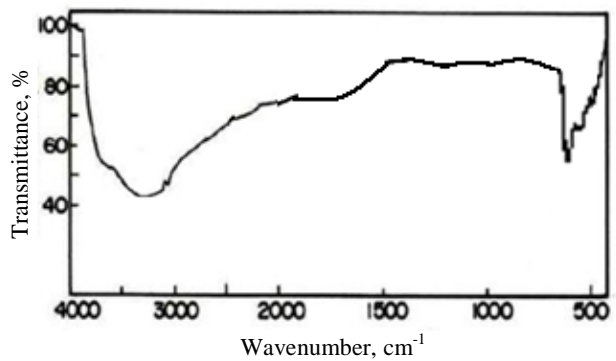

Figure 1. FT-IR of copper oxide nanofluid

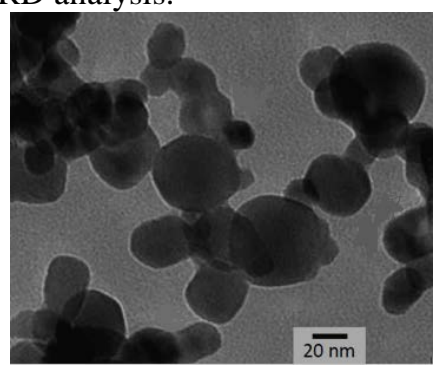

Figure 2. TEM image of copper oxide nanofluid

\section{Elemental dispersive spectroscopy}

The EDS spectroscopy showed the presence of copper and oxygen in the synthesized copper oxide nanofluids by the distinct peaks as observed in the EDS spectrum shown in Figure 3.

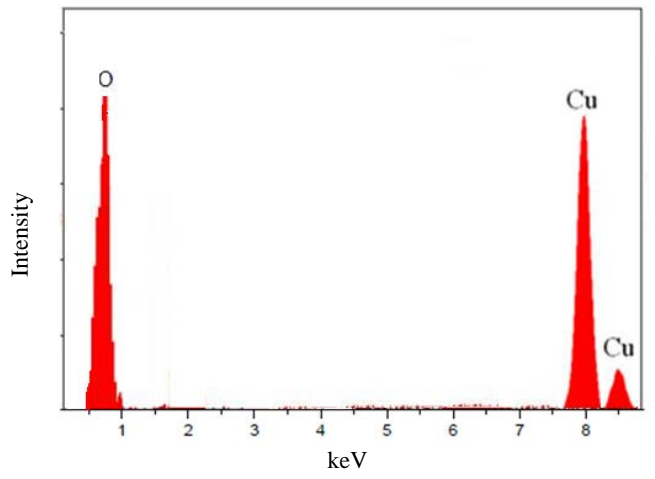

Figure 3. EDS image of copper oxide nanofluid 


\section{$X R D$ analysis}

The XRD results of the copper oxide nanoparticles is shown in Figure 4. The XRD analysis for the crystalline phase of copper oxide $(\mathrm{CuO})$ can be seen. The crystalline phase was determined by x-ray Diffraction (XRD, MACMXP18). All peaks obtained by XRD analysis were assigned by comparison with data from the Joint Committee on powder Diffraction Standards (JCPDS).

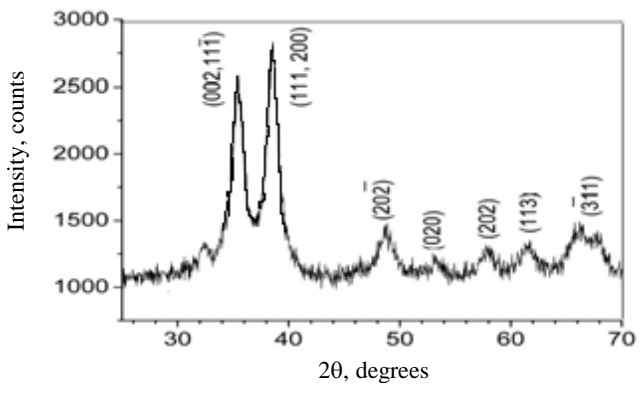

Figure 4. XRD image of copper oxide nanofluid

The XRD image of copper oxide nanopowder (Figure 4) exhibits a single-phase with a monoclinic structure. Lattice parameters are $a=4.84 \AA, \mathrm{b}=3.47 \AA, c=5.33 \AA$. The intensities and positions of peaks are in good agreement with the reported values (JCPDS file No. 05-661). No peaks of impurities are found in XRD pattern. The peaks are broad due to the nano-size effect. The average crystallite size of $\mathrm{CuO}$ nanoparticles is found to be $8 \mathrm{~nm}$ using Scherrer formula.

\section{Measurement of thermal conductivity}

Thermal conductivity can be measured by thermal property analyzer data calculated by experiment is mentioned in Table 1, 2 and 3. The effective thermal conductivity can be measured by thermal property analyzer such as KD2 Pro at different ranges of temperature. The experimental results show that a dramatic increase in the enhancement of thermal conductivity of $\mathrm{CuO}$. This is due to the fact that as the temperature increases, the rate at which $\mathrm{CuO}$ particles moving in the nanofluid increases. The average kinetic energy or energy of motion of the nanoparticles increases with temperature and hence the rate at which heat is transferred in the nanofluid also increases ${ }^{7}$.

Table 1. Values of thermal conductivity at different temperatures for $1 \% \mathrm{CuO}$

\begin{tabular}{ccc}
\hline S.No. & Temperature, ${ }^{\circ} \mathrm{C}$ & Thermal conductivity $(\mathrm{W} / \mathrm{m} / \mathrm{K})$ \\
\hline 1 & 20 & 0.510 \\
2 & 40 & 0.589 \\
3 & 60 & 0.711 \\
4 & 80 & 0.828 \\
\hline
\end{tabular}

Table 2. Values of thermal conductivity at different temperatures for $3 \% \mathrm{CuO}$

\begin{tabular}{ccc}
\hline S.No. & Temperature, ${ }^{\circ} \mathrm{C}$ & Thermal conductivity $(\mathrm{W} / \mathrm{m} / \mathrm{K})$ \\
\hline 1 & 20 & 0.531 \\
2 & 40 & 0.594 \\
3 & 60 & 0.730 \\
4 & 80 & 0.852 \\
\hline
\end{tabular}


Table 3. Values of thermal conductivity at different temperatures for $5 \% \mathrm{CuO}$

\begin{tabular}{ccc}
\hline S.No. & Temperature, ${ }^{\circ} \mathrm{C}$ & Thermal conductivity $(\mathrm{W} / \mathrm{m} / \mathrm{K})$ \\
\hline 1 & 20 & 0.558 \\
2 & 40 & 0.619 \\
3 & 60 & 0.795 \\
4 & 80 & 0.900 \\
\hline
\end{tabular}

\section{Measurement of viscosity}

Viscosity can be measured by Ostwald viscometer at various ranges of temperatures such as $20^{\circ} \mathrm{C}, 40{ }^{\circ} \mathrm{C}, 60{ }^{\circ} \mathrm{C}$ and $80^{\circ} \mathrm{C}$. The final experimental values which are drawn by the experiment are mentioned in Table 4. From the experimental results, it is observed that nanofluids have lower viscosity and higher thermal conductivity. Viscosity was found to decreases with increases in temperature. As temperature increases, the average speed of the molecules in the nanofluid increases and the amount of time the nanoparticles dispersed in the base fluid spend "in contact" with their nearest neighbors decreases. Thus, as temperature increases, the average intermolecular forces decrease in between the nanoparticles. These properties are also depending upon the nanoparticle volume concentration present in the base fluid. Viscosity increases by increasing the particle volume concentration of nanofluids ${ }^{3}$.

Table 4. Viscosity at different temperatures for $1 \%$, $3 \%$ and $5 \% \mathrm{Al}_{2} \mathrm{O}_{3}$

\begin{tabular}{ccccc}
\hline S.No. & Temperature, ${ }^{\circ} \mathrm{C}$ & $\begin{array}{c}\text { Viscosity, (Cp ) } \\
1 \% \mathrm{CuO}\end{array}$ & $\begin{array}{c}\text { Viscosity, (Cp ) } \\
3 \% \mathrm{CuO}\end{array}$ & $\begin{array}{c}\text { Viscosity, (Cp ) } \\
5 \% \mathrm{CuO}\end{array}$ \\
\hline 1 & 20 & 0.6126 & 0.6512 & 0.6983 \\
2 & 40 & 0.4932 & 0.5210 & 0.5837 \\
3 & 60 & 0.1780 & 0.1970 & 0.2204 \\
4 & 80 & 0.0590 & 0.0598 & 0.0839 \\
\hline
\end{tabular}

\section{Conclusion}

In the current research study, three different concentrations of $1 \%, 3 \%$ and $5 \%$ of copper oxide nanofluids were synthesized. These synthesized nanofluids were characterized by FTIR, TEM, EDS and XRD studies. Thermo-physical properties (thermal conductivity and viscosity) measurements were studied.

From the FT-IR analysis of the synthesized nanofluids the structure of the copper oxide nanofluids was ascertained. TEM analysis was carried out to study the morphology of the copper oxide nanoparticles. It was observed that the copper oxide particles are spherical in shape. The chemical composition was studied by EDX and this showed the presence of copper and oxygen. The XRD analysis carried out on the copper oxide nanoparticles showed the crystalline nanometric phases of copper oxide nanoparticles. Thermo-physical properties (thermal conductivity and viscosity) measurements were carried out and it was observed that among the three different nanofluids prepared, 5\% copper oxide containing nanofluids showed the maximum conductivity and the conductivity was found to increase with temperature. The viscosity studies carried out on the three different concentrations of the nanofluids showed that the viscosity was found to decreases with increases in temperature. These properties are also dependent upon the nanoparticle volume concentration present in the base fluid. Viscosity was found to decrease by increasing the particle volume concentration of nanofluids. Hence it could be concluded that the copper oxide nanofluids prepared can be effectively used as coolants for automobile and heat transfer applications. 


\section{References}

1. $\quad$ Pradeep Jaya Sudhan E and Shree Meenakshi K, Ind J Sci Tech., 2011, 4(4), 417-421.

2. $\quad$ Das S K, Putra N, Thiesen P and Roetzel W, J Heat Transf., 2003, 125(4), 567-574; DOI:10.1115/1.1571080

3. Godson L, Raja B, Mohan Lal D and Wongwises S, Exptl Heat Trans., 2010, 23(4), 317-332

4. Eastman J A, Choi S U S, Li S, Yu W and Thompson L J, Appl Phys Lett., 2000, 78(6), 718-720; DOI”10.1063/1.1341218

5. $\quad$ Xuan Y and Li Q, Int J Heat Fluid Flow, 2000, 21(1), 58-64; DOI:10.1016/S0142727X(99)00067-3

6. $\quad$ Kwak K and Kim C, Korea-Aust Rheol J., 2005, 17, 35-40.

7. $\quad$ Duangthongsuk W and Wongwises S, Exptl Thermal Fluid Sci., 2009, 33(4), 706-714; DOI:10.1016/j.expthermflusci.2009.01.005 